\title{
Multiple Epiphyseal Dysplasia (MED): A Rare Type of Skeletal Dysplasia
}

\author{
Mohammad Imnul Islam ${ }^{1}$, Suraiya Begum ${ }^{1}$, Manik Kumar Talukdar ${ }^{1}$, Md Enayet Karim², Shahana Akhter Rahman ${ }^{5}$ \\ ${ }^{1}$ Assistant Professor, ${ }^{3}$ Professor, Department of paediatrics, ${ }^{2}$ Professor, Department of Radiology \& Imaging, Bangabandhu Sheikh Mujib \\ Medical University, Dhaka,
}

\begin{abstract}
:
Multiple epiphyseal dysplasia (MED) is a congenital disorder of skeletal development that primarily affects the ends of long bones, causing progressive joint and bone inflammation and short stature. Mutations in several genes are responsible for pathogenesis of this disease. We are reporting a case of MED who presented with the complaints of multiple swelling of the joints which was associated with pain during movement for last seven years. The patient had flexion deformity of all the affected joints along with restriction of movement. These were associated with kyphosis, pectus carnitum, knock-knee and short stature. Radiological findings were suggestive of MED. Counseling was done with the parents regarding the etiology, progression and outcome of the disease.
\end{abstract}

Keyword: Multiple epiphyseal dysplasia, Flexion deformity.

[BSMMU J 2012; 5(1): 57-60]

\section{Introduction:}

Multiple epiphyseal dysplasia (MED) is a rare genetic disorder of skeletal development which affects the growing ends of bones causing progressive joint and bone inflammation. ${ }^{1}$ Bones usually elongate by a process that involves the deposition of cartilage at the ends of the bones. This cartilage then mineralizes and hardens to become bone. In MED, this process is defective. ${ }^{2}$ Joint pain, joint deformity, waddling gait and short stature are the main clinical signs and symptoms. ${ }^{3}$ Mutations in several genes are responsible for pathogenesis of this disease. Most autosomal forms of MED are attributed to a COMP (cartilage oligomeric matrix protein) mutation and all recessive forms are related to mutations in $S L C 26 A 2$ and involve the peripheral joints. ${ }^{4} \mathrm{We}$ are reporting a case of MED for proper understanding of the problem and to avoid wrong diagnosis and management.

\section{Case report:}

A 14 years old boy, $2^{\text {nd }}$ issue of a consanguineous parents was admitted in the department of paediatrics, Bangabandhu Sheikh Mujib Medical University (BSMMU) with the complaints of multiple swelling of the joints which was associated with pain during movement for last seven years. Initially small joints of hands and feet were involved followed by large joints of the extremities. Pain was non migratory in nature and was not associated with morning stiffness. There was no history of fever, rash, chest pain,

Address for Correspondence: Dr. Mohammad Imnul Islam, Assistant Professor, Department of Paediatrics, Bangabandhu Sheikh Mujib Medical University, Dhaka. Mobile-01711393049, E-mail: imnu127@yahoo.com oral ulcer, photosensitivity or urinary complaints. With these complaints he was treated by local doctors and traditional healers for 3 to 4 years with no improvement. Three years back, he was admitted in a medical college hospital and was diagnosed as a case of JIA and treated with oral Methotrexate and Naproxen for several weeks, but the condition did not improve. Rather gradually the deformites increased.

The boy was delivered normally at term and perinatal period was uneventful. Developmental milestones were normal. He had one younger sister, who was also suffering from similar illness. Their parents were healthy.

On examination the boy was well alert, co-operative and vitals were normal. He had kyposis, pectus carnitum and knock-knee. His weight was $28 \mathrm{~kg}$ (WAZ: -3) and height was $131 \mathrm{~cm}$ (HAZ: -4). He had swelling of both knee, ankle, wrist, elbow and interphalangeal joints of the hands and feet (Fig-1). There was no periarticular wasting of muscle. Colour and temperature of the affected joints were normal. There was no tenderness in the affected joints. Flexion deformities were present in all the affected joints. Movement was restricted in all the affected joints and there was pain during movement. He also had waddling gait.

Routine blood including complete blood count and ESR and urine examination was normal. Serum calcium, inorganic phosphate and alkaline phosphatase were found within normal limit. X-ray of pelvis showed bilateral symmetrical flattening and irregularities of femoral head 


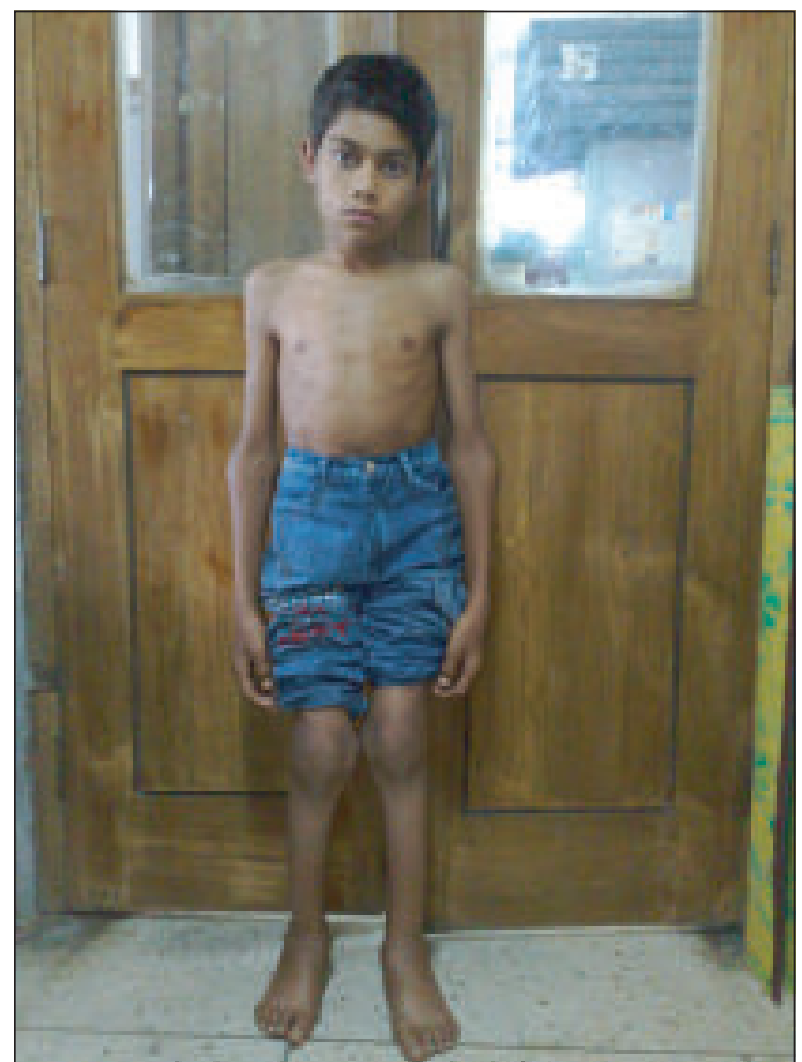

Fig.-1: Showing swelling of both knee, ankle, wrist, elbow and interphalangeal joints of the hands and feet.

with short and broad neck of femur (Fig.-2). Distal epiphyseal end of the proximal interphalangeal joints of $2^{\text {nd }}, 3^{\text {rd }}$ and $4^{\text {th }}$ fingers were expanded (Fig.-3).

$\mathrm{X}$-ray of elbow and ankle joints showed features of osteopenia. X-ray of the dorso-lumber spine showed increased gap between vertebral bodies. Beaking of

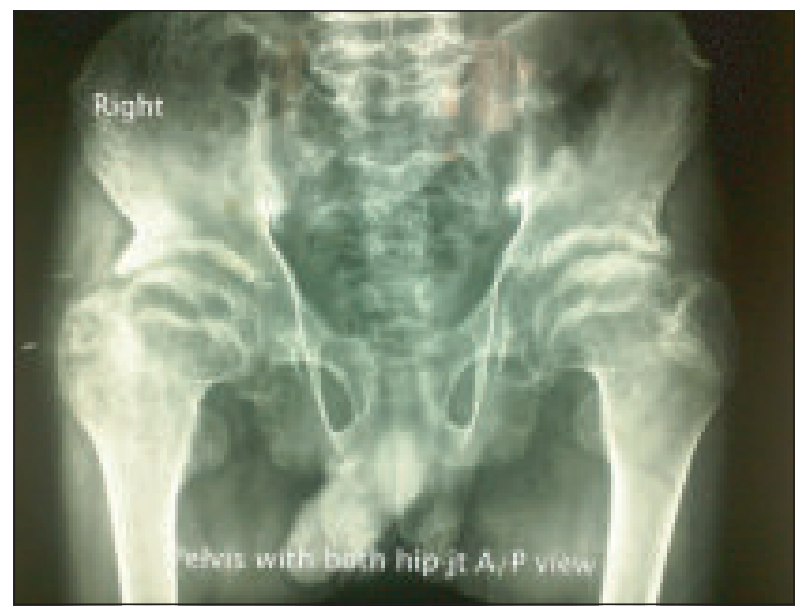

Fig.-2: X-ray Pelvis with hip joint found bilateral symmetrical flattening and irregularities of femoral head with short and broad neck of femur.

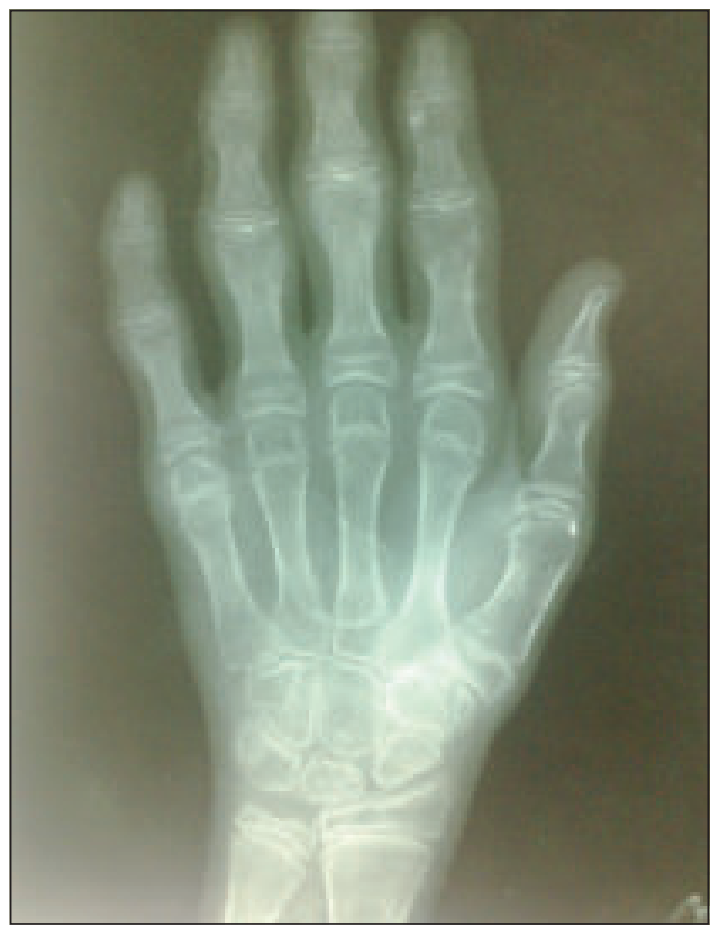

Fig.-3: X-ray hand including wrist joint showing Distal epiphyseal end of the proximal interphalangeal joints of $2^{\text {nd }}, 3^{\text {rd }}$ and $4^{\text {th }}$ fingers were expanded

vertebral bodies and flattened vertebral bodies was also present in our case (Fig.-4).

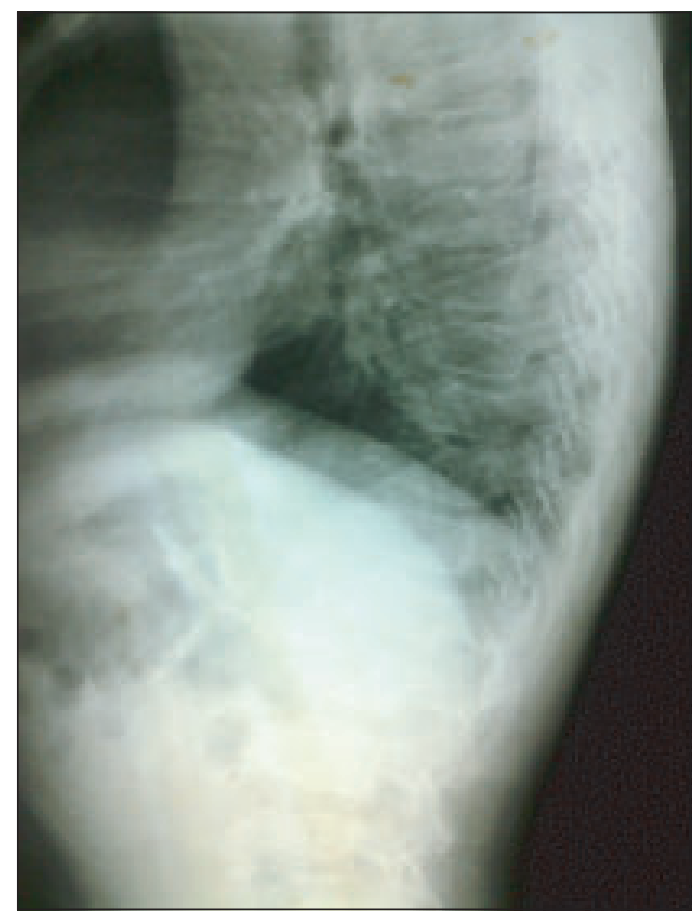

Fig.-4: X-ray dorsolumber spine showing anterior vertebral beaking and increased gap between vertebral bodies. 
Considering history, clinical examinations and investigations this patient was diagnosed as a case of MED. Counseling was done with the parents regarding the etiology, progression and outcome of the disease. Physiotherapy was advised and he was referred to orthopedic surgeon for opinion. He was discharged with the advice for regular follow-up.

\section{Discussion:}

Among the osteochondrodysplasias, multiple epiphyseal dysplasia (MED) occurs most commonly. MED is characterized by involvement of multiple epiphyses with variable phenotypes. MED can be caused by mutations in at least six separate gene. These important genes are: COMP (cartilage oligomeric matrix protein), collagen IX, matrilin 3 and the DTDST or SLC26A2, which encodes the diastrophic dysplasia sulfate transporter. ${ }^{3}$ In general, MED is inherited as an autosomal dominant pattern; other inheritance forms like autosomal recessive pattern are also found. ${ }^{5}$ Studies suggest a prevalence of 9-16 cases per 100,000 births. The exact etiology of MED remains unclear. No potential causes or risk factors for MED are identified. Genetic alterations result in abnormal endochondral ossification. ${ }^{6}$ Altered endochondral ossification may be associated with changes in the articular cartilage. The resultant articular cartilage is deficient in underlying osseous support and fails to withstand normal cyclical loading. Ultimately flexion deformities and contractures of all the affected joints develop. ${ }^{7}$

Variability in the phenotypes of MED may lead to variable presentations. Mild forms of the disease may remain undiagnosed, or they may be misdiagnosed. Presentation may be early or late in childhood.

Patients with MED typically present with some or all of the following features:

- Pain in the hips and/or knees with early fatigue and waddling gait abnormalities.

- Flexion contractures of elbow and angular deformities in the lower extremity like coxa vara, genu varum or valgum. ${ }^{8}$

The height of patients with MED is normal or less than normal. The spine is usually normal in MED, though scoliosis has been reported. Kyphosis also existed in few series. $^{2}$ Our patient had flexion deformities of small and large joints, genu vulgum and waddling gait.

The initial diagnosis of MED is typically based on clinical and radiographic features. Molecular genetic testing has a role in confirming the clinical diagnosis and in establishing a prenatal diagnosis. Radiographs of major joint areas may show small, fragmented, and irregular epiphysis. ${ }^{3}$ The hip radiograph findings are: symmetrical flattening of the epiphysis of the femoral heads. At the same time femoral neck tends to become broad and short .The shoulder radiograph shows symmetrical flattening of the epiphyses of the humeral heads. The ankle joints shows flattening of the epiphyses of the lower end of the tibia, more on the latetral aspect. ${ }^{9}$ In the hand x-ray the phalanges tend to be broad and short. ${ }^{2}$ Anterior vertebral beaking and increased gap between vertebral bodies may be found in $\mathrm{x}$-ray spine. Vertebral bodies can also be found as flattened. The presence of anterior vertebral beaking is classically seen only in Muco Poly Saccharidosis (MPS) and SED (Spondylo epiphyseal dysplasia). Although anterior beaking was seen in our patient, the absence of platyspondyly (decreased gap between vertebral bodies) and presence of irregularities in the vertebral end plates ruled out both SED and Mucopolysaccharidosis (MPS). ${ }^{10}$ The boy had joint deformity and contractures of the affected joints. He also had a affected sister with milder presentations. The radiological findings were consistent with documented findings.

The goals of medical management are to relief pain and halt joint destruction and to prevent early osteoarthritis. Physical therapy program with adequate analgesia is important for pain management. Orthopedic surgery is useful for pain relief, correction of angular deformities, and correction of joint contractures. ${ }^{11}$ We started physiotherapy and also taken opinion regarding corrective surgery from orthopedic surgeons.

Quite a number of children with MED are misdiagnosed as a case of JIA and are given treatment for that. Similar thing happened to our patient as well. In MED features of arthritis are absent which is essential for the diagnosis of JIA. Laboratory evidences like leucocytosis and high ESR are also absent in MED.

\section{Conclusions:}

MED is generalized skeletal disorder associated with significant morbidity. Early diagnosis and initiation of treatment is very much important to prevent joint deformities and contractures. Paediatricians should be cautious about correct diagnosis of this disease. Meticulous history, examination and radiological investigations can confirm the diagnosis.

\section{References:}

1. Multiple Epiphyseal Dysplasia. Wikepedia $13^{\text {th }}$ March, 2011. Available from: http://en.wikipedia.org/wiki/Multiple_ epiphyseal_dysplasia 
2. Ballhausen D, Bonafe L, Terhal P, Unger SL, Bellus G, Classen M. et al. Recessive Multiple Epiphyseal Dysplasia (rMED): phenotype delineation in eighteen homozygotes for DTDST mutation R279W. J Med Genet 2003; 40: 65-71.

3. Zank1 A,Jackson GC,Crettol LM, Taylor J, Elles R, Mortier GR. et al. Preselection of cases through expert clinical and radiological review significantly increases mutation detection rate in Multiple Epiphyseal Dysplasia. Eur J Hum Genet 2007; 15: 150- 4 .

4. Ashish S R, James J M. Multiple Epiphyseal Dysplasia. Emedicine [updated 2009, july,26]. Available from:http:// emedicine. medscape.com/article/1180822-overview.

5. Morrissy RT. Lovell and Winter's Pediatric Orthopaedics. $6^{\text {th }}$ ed. Philadelphia, Pa: Lippincott: Williams and Wilkins; 2006:233-4
6. Wynne-Davies R, Gormley J. The prevalence of skeletal dysplasias: An estimate of their minimum frequency and the number of patients requiring orthopaedic care. J Bone Joint Surg Br 1985; 67:113-7.

7. Stanescu R, Stanescu V, Muriel MP, Maroteaux P. Multiple Epiphyseal Dysplasia, Fairbank type: morphologic and biochemical study of cartilage. Am J Med Genet 1993; 45:501-7.

8. Ingram RR. The shoulder in Multiple Epiphyseal Dysplasia. J Bone Joint Surg Br 1991; 73: 277-9.

9. Yadev P, Narula M. Dysplasia Epiphysealis Multiplex. Indian Journal of Radiol 2000; 10: 267-268.

10. Grover SB, Midha N, Gupta M, Uppal PS. Epiphyseal Dysplasis Tarda. Ind J Radiol Imag 2003; 13: 115-8.

11. Pavone V, Costarella L, Privitera V, Sessa G. Bilateral Total Hip Arthroplasty in Subjects with Multiple Epiphyseal Dysplasia. J Arthroplasty 2009; 24:868-72. 